\title{
The burden of breast cancer in Italy: mastectomies and quadrantectomies performed between 2001 and 2008 based on nationwide hospital discharge records
}

Prisco Piscitelli', Maddalena Barba ${ }^{2}$, Massimo Crespi $^{3}$, Massimo Di Maio $^{4}$, Antonio Santoriello ${ }^{5}$, Massiliamo D’Aiuto ${ }^{6}$, Alfredo Fucito ${ }^{6,7}$, Arturo Losco ${ }^{8}$, Francesca Pentimalli ${ }^{9}$, Pasquale Maranta ${ }^{7,10}$, Giovanna Chitano ${ }^{11}$, Alberto Argentiero ${ }^{11}$, Cosimo Neglia ${ }^{11}$, Alessandro Distante ${ }^{11}$, Gian luca Di Tanna ${ }^{12}$, Maria Luisa Brandi ${ }^{1}$, Alfredo Mazza ${ }^{7}$, Ignazio R Marino ${ }^{13}$, Antonio Giordano ${ }^{7,9,10,14^{*}}$ on behalf of the Human Health Foundation Study Group, in memory of Prof. Giovan Giacomo Giordano

\begin{abstract}
Background: Where population coverage is limited, the exclusive use of Cancer Registries might limit ascertainment of incident cancer cases. We explored the potentials of Nationwide hospital discharge records (NHDRs) to capture incident breast cancer cases in Italy.

Methods: We analyzed NHDRs for mastectomies and quadrantectomies performed between 2001 and 2008. The average annual percentage change (AAPC) and related 95\% Confidence Interval (CI) in the actual number of mastectomies and quadrantectomies performed during the study period were computed for the full sample and for subgroups defined by age, surgical procedure, macro-area and singular Region. Re-admissions of the same patients were separately presented.

Results: The overall number of mastectomies decreased, with an AAPC of $-2.1 \%(-2.3-1.8)$. This result was largely driven by the values observed for women in the 45 to 64 and 65 to 74 age subgroups $(-3.0 \%,-3.4-3.6$ and $-3.3 \%$, $-3.8-2.8$, respectively). We observed no significant reduction in mastectomies for women in the remaining age groups. Quadrantectomies showed an overall +4.7 AAPC (95\%Cl:4.5-4.9), with no substantial differences by age. Analyses by geographical area showed a remarkable decrease in mastectomies, with inter-regional discrepancies possibly depending upon variability in mammography screening coverage and adherence. Quadrantectomies significantly increased, with Southern Regions presenting the highest average rates. Data on repeat admissions within a year revealed a total number of 46,610 major breast surgeries between 2001 and 2008, with an overall $+3.2 \%$ AAPC (95\%Cl:2.8-3.6).
\end{abstract}

Conclusions: In Italy, NHDRs might represent a valuable supplemental data source to integrate Cancer Registries in cancer surveillance.

Keywords: Hospital discharge records, Breast cancer, Mastectomies, Quadrantectomies, Cancer surveillance

\footnotetext{
* Correspondence: giordano@temple.edu

${ }^{7}$ Sbarro Institute for Cancer Research and Molecular Medicine and Center of Biotechnology, College of Science and Technology Temple University, BioLife Science, Bldg. Suite 4311900 N 12th Street, Philadelphia, PA 19122, USA ${ }^{9}$ INT-CROM, National Cancer Institute G. Pascale Foundation - Cancer Research Center, Via Ammiraglio Bianco, Mercogliano, Avellino 83013, Italy Full list of author information is available at the end of the article
} 


\section{Background}

Cancer incidence data are a cornerstone of epidemiology research, health monitoring and resource allocation for interventions aimed at cancer prevention and control. Cancer Registries (CRs) contribute to cancer surveillance at local level, throughout the process of systematic collection of data about the occurrence and characteristics of reportable neoplasms [1]. In United States, the National cancer statistics are built on data from a network of CRs called the Surveillance, Epidemiology and End Results Program (SEER). The SEER has now expanded its coverage to $26 \%$ of the total population of the United States, accounting for 65.4 million people. Registries included in the SEER share requirements in data reporting and verification procedures throughout a quality improvement process restructured in year 2000. However, the exclusive use of CRs poses limits to the nationwide ascertainment of incident cancer cases, with major concerns arising from the percentage of US population still uncovered [2].

Various secondary databases have been proposed as potential tools to enhance the detection of incident cases and related treatments for a number of diseases, including cancer [3-6]. The accuracy of secondary data sources in capturing cases has been explored with results varying upon the source selected and gold standard used [6-9]. In the study from Penberthy et al., the Virginia Cancer Registry (CR) and a statewide hospital discharge file (HDF) were both tested for accuracy in correctly identifying a cancer and its site of origin. Data from inpatient medical records were used as the gold standard. Based on the conclusions stated, nor the CR neither the HDF was sufficient independently to allow the complete capture of incident cancer cases. However, HDF accuracy in capturing incident cancer cases was high, with the overall positive predictive value being $94 \%$ and site specific values ranging from $86 \%$ (cervix) to $98 \%$ (breast) [9]. In Italy, the government supports cancer surveillance throughout a network of population-based local CRs included in the Italian Association of Cancer Registries (AIRTUM). Currently, the AIRTUM covers $33.8 \%$ of the Italian population, namely 19 million people out of 61 million inhabitants. A notable disproportion in CRs coverage exists among Northern, Central and Southern areas of Italy (i.e., $50.2 \%, 25.5 \%$ and $17.9 \%$, respectively) [10].

We have previously underlined the need to integrate data from the Italian CRs with additional sources and identified the National Hospital Discharge Records (NHDRs) as a potential tool [11].

In this study we aimed to evaluate the burden of breast cancer in Italian women by analyzing data from the NHDRs through a non-model-based methodology with a specific focus on major surgical procedures.
Compared to our previous work, data have been updated to reflect a larger time window (2001-2008 vs. 20002005) and methods refined to overcome some of the limitations from our previous study.

\section{Materials and methods \\ Data source}

We used the NHDR database which includes records from all the Italian public and private hospitals. Data were made available by the Italian Ministry of Health relatively to the time frame between 2001 and 2008 . These data were subject to a systematic quality assessment performed at a Regional and central level. The matching with the National Institute for Statistics (ISTAT) by social security code showed a percentage of correct linkage increasing from $95.6 \%$ in $2001(50,921$ records matched out of 53,226$)$ to $99.8 \%$ in 2008 (58,367 records matched out of 58,492) [12,13]. The years 1999 and 2000 were excluded due to incomplete data.

Breast cancer cases were identified on the basis of the International Classification of Diseases, Ninth Revision, Clinical Modification (ICD-9-CM) [14,15]. We considered patients diagnosed with invasive breast cancer (i.e., malignant neoplasm of breast, ICD-9CM codes: 174.0-174.9 and 175.0-175.9). Data related to patients with in situ breast carcinoma (ICD-9-CM major diagnosis 233) were also included.

\section{Population}

Eligible women were patients diagnosed with incident, histologically-confirmed breast cancer who underwent major breast surgical procedures between 2001 and 2008, as identified based on the following codes from the ICD-9-CM: 85.41-48 (mastectomies), 85.22 (quadrantectomies), 85.23 (subtotal mastectomies) [14,15]. In data analysis, mastectomies and subtotal mastectomies (ICD-9-CM codes: $85.41-48$ and 85.23 , respectively) were ascribed to the same category of major breast surgery (i.e., mastectomies). Excision biopsies and tumorectomies (ICD9-CM code 85.21) were not included. Thus, patients with benign lesions were not considered in our analysis. In order to minimize the overlap between prevalent and incident cases, repeated admissions in any calendar year and across different years for the entire time window considered were discounted and reported separately. We included records pertinent to ordinary hospitalization as well as day hospital regimens.

\section{Statistical analyses}

Data were analyzed using STATA/SE version 11 for Windows (StataCorp LP, College Station, TX, USA) and Microsoft Office Excel 2007 (Microsoft Corp, Seattle, WA, USA). The average annual percentage change (AAPC) and related 95\% Confidence Interval (CI) in the 
actual number of mastectomies and quadrantectomies performed during the study period were computed using a Poisson regression model. To describe time trends, we carried out joinpoint regression analysis.

Analyses were performed for the full sample as well as for subgroups defined by type of surgical procedure (mastectomies and quadrantectomies), age (25-39, 40$44,45-64,65-74$ and $\geq 75$ years old), and geographical area [i.e., Region and macro-areas (Northern, Central and Southern Italy)]. Results by geographical area were presented in a frame including the indicators of extension and adherence to the national breast cancer screening programs [16].

\section{Results}

Mastectomies and quadrantectomies performed in Italy between 2001 and 2008 are reported in Table 1 and Table 2, respectively. The overall number of mastectomies decreased from 15,754 (year 2001) to 14,197 (year 2008), with an AAPC of $-2.1 \%(-2.3-1.8)$. This result is largely driven by the values observed for women in the 45 to 64 and 65 to 74 age subgroups $(-3.0 \%,-3.4-3.6$ and $-3.3 \%,-3.8-2.8$, respectively) and, at a lesser extent, in women aged 75 years and older $(-1.2 \%,-1.7-0.7)$. We observed no significant reduction in mastectomies for women aged $25-39$ years $(+0.3 \% ;-0.8-1.3)$ and $40-44$ years (+1.5\%; 0.5-2.5).

As shown in Table 2, there was a $+4.7 \%$ increase in quadrantectomies $(95 \% \mathrm{CI} 4.5-4.9)$ with the actual numbers rising from 22,140 (year 2001) to 30,800 (year 2008). Temporal trends of mastectomies and quadrantectomies between 2001 and 2008 are shown in Figure 1. Mastectomies were always performed during ordinary hospitalizations, while quadrantectomies performed in a day hospital regimen progressively increased over the 8- year period $(+74.2 \%)$, accounting for more than $17.5 \%$ of the overall breast surgery procedures in 2008 (data available upon request).

In Table 3, we present data by singular Italian Region and macro-areas (i.e., Northern, Central and Southern Italy). Remarkable decreases in the number of mastectomies performed in Italy between 2001 and 2008 were observed in Northern and Central Italy $(-2.7 \%,-3.0-2.4$ and $-2.9 \%,-3.4-2.4$, respectively) but not in Southern Italy $(0.3 \%,-0.3-0.8)$, where statistically significant reductions were reported for Campania, Calabria and Sicily only.

Quadrantectomies significantly increased across all the Regions but Valle D'Aosta and Abruzzo. When macroareas were considered, the most remarkable increase was reported for Southern Regions (+3.3\%, 3.0-3.5; $+3.9 \%, 3.5-4.3$ and $+7.2 \%, 6.8-7.7$ for Northern, Central and Southern regions, respectively).

In Table 4, we report mastectomies and quadrantectomies performed on repeated admissions in the same year between 2001 and 2008. Overall, a total number of 46,610 repeated breast surgeries was performed in Italy between 2001 and 2008. Our data showed a significant increase in any of the subcategories considered but the first one (i.e., subcategory including women who underwent repeated breast surgery once within the same year).

\section{Discussion}

In the present study, data from the NHDRs proved a valuable tool in the ascertainment of the real figures of incident breast cancer cases. Indeed, the current indications for quadrantectomies or mastectomies in operable breast cancer, along with the use of well defined codes assigned to breast surgeries at the time of patient

Table 1 Mastectomies ${ }^{1}$ performed in Italy between 2001 and 2008

\begin{tabular}{|c|c|c|c|c|c|c|c|c|c|c|}
\hline Age-group & 2001 & 2002 & 2003 & 2004 & 2005 & 2006 & 2007 & 2008 & Subtotals & AAPC $(95 \% \mathrm{Cl})^{2}$ \\
\hline \multirow[t]{2}{*}{$25-39$} & 854 & 819 & 849 & 851 & 800 & 786 & 812 & 921 & 6,692 & \\
\hline & & & & & & & & & & $+0.3(-0.8 ; 1.3)$ \\
\hline \multirow[t]{2}{*}{$40-44$} & 907 & 875 & 962 & 957 & 927 & 1008 & 955 & 999 & 7,590 & \\
\hline & & & & & & & & & & $+1.5(0.5 ; 2.5)$ \\
\hline \multirow[t]{2}{*}{$45-64$} & 5849 & 5805 & 5353 & 5251 & 4950 & 4811 & 4783 & 4974 & 41,776 & \\
\hline & & & & & & & & & & $-3.0(-3.4 ;-3.6)$ \\
\hline \multirow[t]{2}{*}{$65-74$} & 3870 & 3802 & 3646 & 3596 & 3310 & 3193 & 3129 & 3178 & 27,724 & \\
\hline & & & & & & & & & & $-3.3(-3.8 ;-2.8)$ \\
\hline \multirow[t]{2}{*}{$75-100$} & 4274 & 4464 & 4516 & 4265 & 4126 & 4157 & 4053 & 4125 & 33,980 & \\
\hline & & & & & & & & & & $-1.2(-1.7 ;-0.7)$ \\
\hline Subtotals & 15,754 & 15,765 & 15,326 & 14,920 & 14,113 & 13,955 & 13,732 & 14,197 & 117,762 & \\
\hline
\end{tabular}

Data are reported by age.

${ }^{1}$ Reported data are absolute numbers unless otherwise specified.

${ }^{2}$ AAPC (95\% Cl): Average Annual Percentage Change and 95\% Confidence Interval. 
Table 2 Quadrantectomies ${ }^{1}$ performed in Italy between 2001 and 2008

\begin{tabular}{|c|c|c|c|c|c|c|c|c|c|c|}
\hline Age group & 2001 & 2002 & 2003 & 2004 & 2005 & 2006 & 2007 & 2008 & Subtotals & AAPC $(95 \% \mathrm{Cl})^{2}$ \\
\hline \multirow[t]{2}{*}{$25-39$} & 1337 & 1375 & 1474 & 1691 & 1722 & 1730 & 1706 & 1650 & 12,685 & \\
\hline & & & & & & & & & & $+3.6(2.8 ; 4.3)$ \\
\hline \multirow[t]{2}{*}{$40-44$} & 1664 & 1839 & 1886 & 2216 & 2296 & 2473 & 2510 & 2610 & 17,494 & \\
\hline & & & & & & & & & & $+6.7(6.0 ; 7.4)$ \\
\hline \multirow[t]{2}{*}{$45-64$} & 11573 & 12032 & 12334 & 12952 & 13294 & 13614 & 13908 & 14820 & 104,527 & \\
\hline & & & & & & & & & & $+3.4(3.1 ; 3.6)$ \\
\hline \multirow[t]{2}{*}{$65-74$} & 5021 & 5331 & 5510 & 5913 & 6048 & 6550 & 6732 & 7154 & 48,259 & \\
\hline & & & & & & & & & & $+5.1(4.7 ; 5.5)$ \\
\hline \multirow[t]{2}{*}{$75-100$} & 2545 & 2912 & 3139 & 3336 & 3624 & 3936 & 4103 & 4566 & 28,161 & \\
\hline & & & & & & & & & & $+8.1(7.5 ; 8.6)$ \\
\hline \multirow[t]{2}{*}{ Subtotals } & 22,140 & 23,489 & 24,343 & 26,108 & 26,984 & 28,303 & 28,959 & 30,800 & 211,126 & \\
\hline & & & & & & & & & & $+4.7(4.5 ; 4.9)$ \\
\hline
\end{tabular}

${ }^{1}$ Reported data are absolute numbers unless otherwise specified.

${ }^{2}$ AAPC: Average Annual Percentage Change and 95\% Confidence Interval.

Data are reported by age groups.

discharge, render breast cancer particularly prone to traceability through NHDRs. Based on our results, mastectomies decreased in all the age groups but two (i.e., women aged 25-39 and 40-44 years). Conversely, quadrantectomies showed a significant increase across all the age groups. There was a significant decrease in the number of mastectomies in Northern and Central Italy but not in Southern Italy, where the inter-regional differences were remarkable. Quadrantectomies significantly increased across all the different Regions (but Valle D'Aosta and Abruzzo) and macro areas considered.
This study has several strengths. Data were made available by the Italian Ministry of Health. Given that the hospital discharge records provide the basis for hospital care reimbursement within a diagnosis-related groups (DRGs) system, these data are subject to a systematic quality assessment performed at a Regional and central level. Dedicated programs and multidisciplinary workgroups are in place at the Department of Quality Assessment, Management of Medical Care and Ethics of the Italian Ministry of Health to enhance data accuracy and completeness. Constant efforts have led to

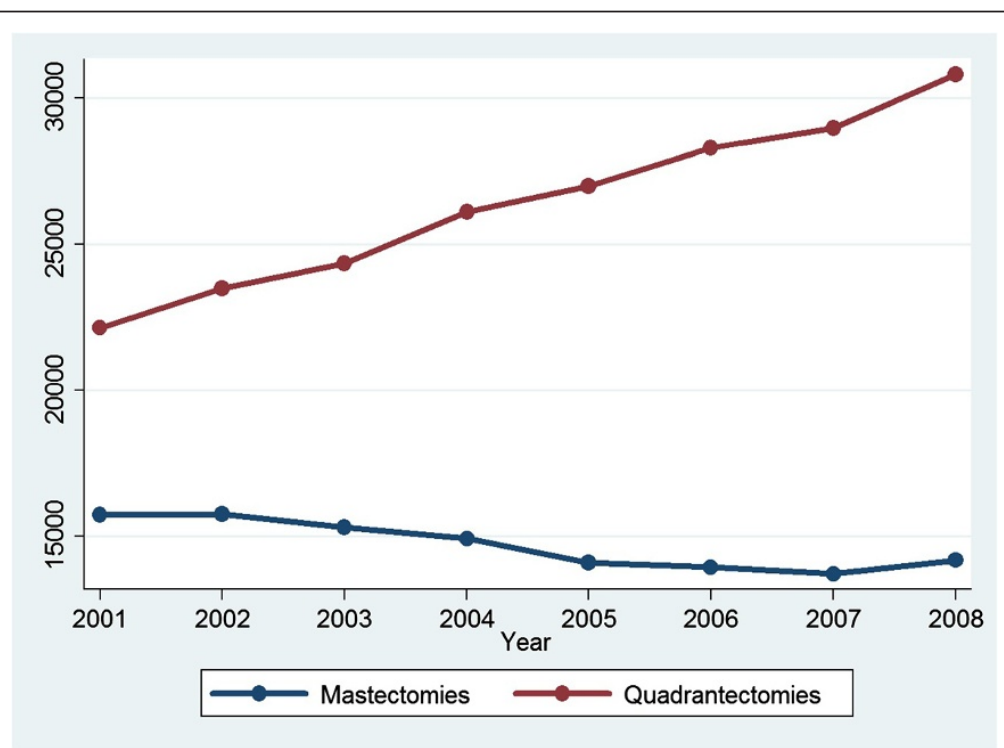

Figure 1 Temporal Trends in Mastectomies and Quadrantectomies performed in Italy between 2001 and 2008. Joinpoint analysis for mastectomies and quadrantectomies (absolute numbers) performed in Italy between 2001-8. 
Table 3 Mastectomies ${ }^{1}$ (Ms) and Quadrantectomies ${ }^{1}$ (Qs) performed in Italy between 2001 and 2008

\begin{tabular}{|c|c|c|c|c|c|c|c|c|c|c|c|}
\hline Region & $\begin{array}{l}\text { Mammographic } \\
\text { screening } \\
\text { coverage }(\%)^{*}\end{array}$ & $\begin{array}{l}\text { Adherence to } \\
\text { mammographic } \\
\text { screening }(\%)^{\S}\end{array}$ & 2001 & 2002 & 2003 & 2004 & 2005 & 2006 & 2007 & 2008 & AAPC $(95 \% \mathrm{Cl})^{2}$ \\
\hline Piemonte Ms & $68.6 \%$ & $65.6 \%$ & 1222 & 1177 & 1138 & 1146 & 1112 & 1140 & 1053 & 1032 & $-2.1(-2.9 ;-1.2)$ \\
\hline Qs & & & 1686 & 1636 & 1714 & 1856 & 1881 & 2024 & 2160 & 2268 & $+4.9(4.2 ; 5.6)$ \\
\hline Valle d'Aosta Ms & $92,3 \%$ & $79,0 \%$ & 35 & 26 & 26 & 28 & 16 & 30 & 24 & 23 & $-4.2(-9.8 ;+1.6)$ \\
\hline Qs & & & 50 & 62 & 64 & 73 & 76 & 77 & 64 & 72 & $+3.7(0.0 ; 7.6)$ \\
\hline Lombardia Ms & $92,8 \%$ & $64,5 \%$ & 3690 & 3511 & 3295 & 3199 & 2985 & 2844 & 2845 & 3063 & $-3.4(-3.9 ;-2.9)$ \\
\hline$\overline{Q s}$ & & & 6257 & 6542 & 6428 & 6667 & 6915 & 7048 & 7245 & 7322 & $+2.3(1.9 ; 2.7)$ \\
\hline P. A. di Bolzano Ms & n.a. & $52,5 \%$ & 122 & 113 & 107 & 110 & 93 & 94 & 95 & 89 & $-4.3(-7.1 ;-1.4)$ \\
\hline Qs & & & 97 & 69 & 70 & 87 & 78 & 142 & 144 & 175 & $+13.5(10.2 ; 17.0)$ \\
\hline P. A. di Trento Ms & $80,9 \%$ & $79,2 \%$ & 115 & 127 & 129 & 128 & 146 & 135 & 119 & 134 & $+1.2(-1.5 ;+3.9)$ \\
\hline Qs & & & 136 & 175 & 166 & 216 & 208 & 236 & 209 & 251 & $+9.4(7.5 ; 11.4)$ \\
\hline Veneto Ms & $76,8 \%$ & $77,1 \%$ & 1512 & 1475 & 1457 & 1267 & 1200 & 1312 & 1305 & 1406 & $-1.8(-2.6 ;-1.0)$ \\
\hline Qs & & & 1510 & 1612 & 1588 & 1674 & 1595 & 1893 & 2075 & 2296 & $+14.7(13.8 ; 15.6)$ \\
\hline Friuli Venezia Giulia Ms & $98,7 \%$ & $62,6 \%$ & 539 & 550 & 571 & 529 & 529 & 534 & 545 & 527 & $-0.5(-1.8 ; 0.8)$ \\
\hline Qs & & & 533 & 526 & 563 & 606 & 710 & 930 & 809 & 798 & $+8.2(6.9 ; 9.4)$ \\
\hline Liguria Ms & $34,4 \%$ & $66,9 \%$ & 405 & 393 & 402 & 376 & 420 & 350 & 301 & 334 & $-3.4(-4.9 ;-1.8)$ \\
\hline Qs & & & 809 & 847 & 893 & 1.010 & 993 & 1.063 & 1049 & 1077 & $+6.2(5.1 ; 7.3)$ \\
\hline Emilia Romagna Ms & $96,0 \%$ & $72,4 \%$ & 1530 & 1542 & 1382 & 1372 & 1200 & 1253 & 1274 & 1262 & $-3.3(-4.1 ;-2.5)$ \\
\hline$\overline{\text { Qs }}$ & & & 2061 & 2169 & 2148 & 2.378 & 2644 & 2690 & 2666 & 2927 & $+5.2(4.6 ; 5.8)$ \\
\hline Total Northern Italy Ms & $82,0 \%$ & $67,9 \%$ & 9,170 & 8,914 & 8,507 & 8,155 & 7,701 & 7,692 & 7,561 & 7,870 & $-2.7(-3.0 ;-2.4)$ \\
\hline Qs & & & 13,139 & 13,638 & 13,634 & 14,567 & 15,100 & 16,103 & 16,421 & 17,186 & $+3.3(3.0 ; 3.5)$ \\
\hline Toscana Ms & $86,4 \%$ & $69,5 \%$ & 968 & 994 & 841 & 853 & 796 & 814 & 845 & 782 & $-3.0(-4.0 ; 2.0)$ \\
\hline Qs & & & 1661 & 1859 & 1871 & 2055 & 1960 & 2037 & 2010 & 2022 & $+2.3(1.6 ; 3.0)$ \\
\hline Umbria Ms & $89,0 \%$ & $73,3 \%$ & 249 & 197 & 195 & 216 & 190 & 179 & 161 & 209 & $-3.1(-5.1 ;-1.0)$ \\
\hline Qs & & & 443 & 429 & 453 & 436 & 471 & 501 & 482 & 550 & $+3.1(1.6 ; 4.5)$ \\
\hline Marche Ms & $74,2 \%$ & $54,2 \%$ & 485 & 515 & 483 & 486 & 472 & 413 & 371 & 378 & $-4.4(-5.7 ;-3.0)$ \\
\hline Qs & & & 482 & 537 & 536 & 587 & 653 & 678 & 731 & 753 & $+6.7(5.4 ; 8.0)$ \\
\hline Lazio Ms & $63,6 \%$ & $47,6 \%$ & 1516 & 1652 & 1456 & 1489 & 1405 & 1382 & 1325 & 1368 & $-2.4(-3.2 ;-1.6)$ \\
\hline Qs & & & 2.222 & 2376 & 2581 & 2771 & 2950 & 2759 & 2849 & 3330 & $+4.9(4.2 ; 5.5)$ \\
\hline \multirow[t]{2}{*}{ Abruzzo Ms } & $56,6 \%$ & $50,5 \%$ & 267 & 270 & 206 & 225 & 219 & 187 & 217 & 236 & $-2.8(-4.7 ;-0.8)$ \\
\hline & & & 381 & 375 & 310 & 376 & 332 & 386 & 424 & 421 & $+2.3(0.7 ; 3.9)$ \\
\hline
\end{tabular}


Table 3 Mastectomies ${ }^{1}$ (Ms) and Quadrantectomies' (Qs) performed in Italy between 2001 and 2008 (Continued)

\begin{tabular}{|c|c|c|c|c|c|c|c|c|c|c|c|}
\hline Total Central Italy Ms & $78,5 \%$ & $59,7 \%$ & 3,485 & 3,628 & 3,181 & 3,269 & 3,082 & 2,975 & 2,919 & 2,973 & $-2.9(-3.4 ;-2.4)$ \\
\hline Qs & & & 5,189 & 5,576 & 5,751 & 6,225 & 6,366 & 6,361 & 6,496 & 7,076 & $+3.9(3.5 ; 4.3)$ \\
\hline Molise Ms & $48,5 \%$ & $43,4 \%$ & 62 & 55 & 83 & 74 & 69 & 63 & 76 & 47 & $-1.2(-4.8 ;+2.6)$ \\
\hline Qs & & & 46 & 70 & 83 & 117 & 103 & 115 & 95 & 121 & +9.8 (6.4; 13.4) \\
\hline Campania Ms & $50,0 \%$ & $29,6 \%$ & 897 & 909 & 950 & 968 & 878 & 786 & 813 & 797 & $-2.4(-3.4 ;-1.4)$ \\
\hline Qs & & & 1.194 & 1271 & 1323 & 1429 & 1495 & 1568 & 1687 & 1885 & $+6.4(5.6 ; 7.3)$ \\
\hline Puglia Ms & $25,3 \%$ & $33,4 \%$ & 987 & 928 & 903 & 933 & 901 & 963 & 959 & 1051 & $+0.9(0.0 ; 1.9)$ \\
\hline Qs & & & 1.010 & 1174 & 1182 & 1315 & 1324 & 1361 & 1410 & 1520 & $+12.8(11.7 ; 13.8)$ \\
\hline Basilicata Ms & $100,0 \%$ & $49,2 \%$ & 88 & 98 & 78 & 75 & 89 & 110 & 107 & 114 & $+4.3(1.1 ; 7.6)$ \\
\hline Qs & & & 81 & 59 & 92 & 97 & 99 & 110 & 112 & 135 & $+8.9(5.6 ; 12.3)$ \\
\hline Calabria Ms & $51,8 \%$ & $26,2 \%$ & 295 & 322 & 320 & 287 & 237 & 239 & 245 & 221 & $-5.1(-6.9 ;-3.4)$ \\
\hline Qs & & & 195 & 225 & 233 & 302 & 355 & 380 & 362 & 434 & $+11.7(9.8 ; 13.7)$ \\
\hline Sicilia Ms & $49,2 \%$ & $41,7 \%$ & 770 & 911 & 856 & 743 & 724 & 719 & 654 & 696 & $-3.4(-4.5 ;-2.4)$ \\
\hline Qs & & & 1.286 & 1476 & 1616 & 1542 & 1691 & 1819 & 1765 & 1846 & $+4.6(3.8 ; 5.4)$ \\
\hline Sardegna Ms & $57,2 \%$ & $54,1 \%$ & - & - & 448 & 416 & 432 & 408 & 398 & 428 & $-1.1(-3.4 ;+1.1)$ \\
\hline Qs & & & - & - & 429 & 514 & 451 & 486 & 611 & 597 & $+6.7(4.5 ; 8.9)$ \\
\hline Total Southern Italy Ms & $46,5 \%$ & $36,3 \%$ & 3,099 & 3,223 & 3,638 & 3,496 & 3,330 & 3,288 & 3,252 & 3,354 & $+0.3(-0.3 ;+0.8)$ \\
\hline$Q s$ & & & 3,812 & 4,275 & 4,958 & 5,316 & 5,518 & 5,839 & 6,042 & 6,538 & $+7.2(6.8 ; 7.7)$ \\
\hline Subtotal ITALY Ms & $72,7 \%$ & $60,0 \%$ & 15,754 & 15,765 & 15,326 & 14,920 & 14,113 & 13,955 & 13,732 & 14,197 & $-2.1(-2.3 ;-1.8)$ \\
\hline Qs & & & 22,140 & 23,489 & 24,343 & 26,108 & 26,984 & 28,303 & 28,959 & 30,800 & $+12.9(12.7 ; 13.2)$ \\
\hline Total ITALY Ms + Qs & & & 37,894 & 39,254 & 39,669 & 41,028 & 41,097 & 42,258 & 42,691 & 44,997 & $+2.2(2.0 ; 2.3)$ \\
\hline
\end{tabular}

Data are reported by region and macro-area (Northern, Central, and Southern Italy).

${ }^{1}$ Reported data are absolute numbers unless otherwise specified.

${ }^{2}$ AAPC: Average Annual Percentage Change and 95\% Confidence Interval.

*Percentage of women aged 50-69 years old (on total screening target population) invited to perform mammographic screening in $2007-2008$ (2-year cumulative data). ${ }^{18} \S$ Adherence rate to mammography screening in year 2008 (adjusted by excluding women performing mammography outside official programs). ${ }^{16}$

Percentages of coverage and adherence to mammographic screening in 2007-08 are also reported. ${ }^{16}$ 
Table $4{ }^{1}$ Mastectomies and ${ }^{1}$ Quadrantectomies performed on repeated admissions between 2001 and 2008

\begin{tabular}{lccccccccc}
\hline Re-interventions $(\mathbf{n})$ in the same patient & $\mathbf{2 0 0 1}$ & $\mathbf{2 0 0 2}$ & $\mathbf{2 0 0 3}$ & $\mathbf{2 0 0 4}$ & $\mathbf{2 0 0 5}$ & $\mathbf{2 0 0 6}$ & $\mathbf{2 0 0 7}$ & $\mathbf{2 0 0 8}$ & AAPC (95\%Cl) \\
\hline $\mathbf{1}$ re-intervention in the same year & 3268 & 3243 & 3241 & 3039 & 2950 & 2667 & 2347 & 1796 & $-6.8(-7.3 ;-6.3)$ \\
$\mathbf{2}$ re-interventions in the same year & 1387 & 1981 & 2419 & 2834 & 3092 & 3484 & 3560 & 3794 & $+12.9(12.2 ; 13.5)$ \\
$\mathbf{3}$ re-interventions in the same year & 27 & 56 & 132 & 166 & 220 & 240 & 290 & 295 & $+27.5(24.4 ; 30.7)$ \\
$>\mathbf{3}$ re-interventions in the same year & 0 & 0 & 7 & 3 & 17 & 16 & 15 & 24 & $++45.9(29.9 ; 63.9)$ \\
Total Re-interventions & 4682 & 5280 & 5799 & 6042 & 6279 & 6407 & $\mathbf{6 2 1 2}$ & 5909 & $+3.2(2.8 ; 3.6)$ \\
\hline
\end{tabular}

Data is presented by categories defined upon the number of repeat major breast surgeries within a year.

${ }^{1}$ Reported data are absolute numbers unless otherwise specified.

${ }^{2}$ AAPC: Average Annual Percentage Change (with 95\% Confidence Interval, CI).

substantial improvements in data quality. Demographic data accuracy was high. However, inter-regional differences in the completeness of reporting exist and must be taken into account when considering these data [12].

We specifically focused on breast cancer patients having undergone mastectomy or quadrantectomy, whose basic requirement is a histologically-confirmed diagnosis of primary breast cancer. At the same time, we excluded women having undergone excision biopsies and tumorectomies. This approach significantly minimized the inclusion of false positive cases.

Repeated admissions were identified and discounted over the entire 8-year period. This increases our confidence in the ability of the NHDRs to differentiate patients with incident breast cancer cases, included in the present study, from patients with prevalent cancers. Data on repeat admissions were approached in a separate set of analyses (Table 4). Future work will be oriented towards the identification of factors associated with surgery-related hospital readmissions in breast cancer patients, with a specific focus on tumor size and histology, lymph node involvement, type of surgical treatment and patient demographics.

In our analysis, we included data on in situ breast carcinoma. The latter accounted for a small average number of major breast surgeries performed on a yearly basis [i.e., 234 mastectomies (range: 227-301), and 1004 quadrantectomies (range: 725-1300) per year]. In situ breast cancer holds the potentials for malignant transformation. The systematic collection, analysis and reporting of data on carcinoma in situ might help identify risk factors and clarify underlying mechanisms of malignant transformation, thus contributing to breast cancer control research and more targeted treatments $[17,18]$.

Our study has also some limitations. Based on predefined selection criteria, our study population includes women eligible for quadrantectomies or mastectomies. The latter category encompasses patients diagnosed with early and locally advanced breast cancer, while generally excluding patients with metastatic breast cancer $(\mathrm{MBC})$ at the time of diagnosis. On this basis, our analysis is expected to underestimate the actual number of breast cancer incident cancer cases. Currently, the percentage of breast cancer patients who are metastatic at diagnosis approximates $6 \%$, with a 5 -year survival rate of $21 \%$ [19].

We analyzed data related to the time frame spanning from 2001 to 2008. Variations in admitting practices and treatment protocols for the disease of interest might have occurred over time and by area. In few cases, this could have caused discrepancies between the hospital discharges and the actual occurrence of the disease considered [20,21].

Notwithstanding the exclusion of incident cases of metastatic breast cancer (by inclusion criteria), the rates obtained from the analysis of the hospital discharge records were higher than those reported by the Italian Ministry of Health in 2006. According to the CRs 2006 report, the number of estimated breast cancer cases for the year 2006 was 37,542 [22]. In the same year, we observed 42,258 cases (i.e., +11\%). Several factors might contribute to such a discrepancy. First, in our study the linking process allowed the discharge of repeat hospital admission between 2001 and 2008, but discharge data related to patients who had been admitted for breast cancer in years prior to 2001 might still be present. Indeed, 10-15 percent of patients undergoing breast conservative therapy for operable breast cancer (i.e., breast-conserving surgery and postoperative breast irradiation) will develop a loco-regional recurrence within 10 years [23]. This risk is slightly higher than that of a loco-regional recurrence following mastectomy (5 to 10 percent) $[23,24]$. However, these rates include both metastases occurring in the ipsilateral preserved breast (i.e., local recurrence) and regional lymph nodes, (i.e., regional recurrence), with only the first representing a potential target for breast surgery. Second, our analysis included data on carcinoma in situ of the breast, which are not routinely collected and analyzed by CRs [17]. Third, the official estimates were based on the use of the Mortality and Incidence Analysis Model method (MIAMOD), a back-calculation approach which obtains cancer-specific morbidity measures by using official mortality data and model-based relative survival from local cancer registry data. As such, the MIAMOD 
method reflects the limitations stemming from the incomplete coverage and disproportion among macroareas which characterize the Italian network of CRs [10]. On this basis, underreporting of cases and, consequently, underestimation of the cancer burden cannot be excluded when using the MIAMOD approach.

Significant increases in quadrantectomies were reported in women aged 25 to 39 and 40 to 44 years. Women in these age groups are still formally uncovered by the breast cancer screening programs activated by the Italian Ministry of Health, despite they represent 13.6\% of women undergoing total major breast procedures [16].

In general, our figures showed inverse trends for mastectomies and quadrantectomies performed in Italy between 2001 and 2008. The increase observed for quadrantectomies and the decrease concerning mastectomies might be interpreted in light of the progressive expansion of the screening programs, and the better adherence to updated treatment protocols [16]. Indeed, mammographic screen-detected cancers show more favorable prognostic features at diagnosis and need less extensive treatment compared to symptomatic cancers [25]. The heterogeneous distribution of such interventions (i.e., screening programs), particularly in Southern Italy, might account for the differences in trends across macro areas and singular regions.

Several studies have investigated the use of hospital discharge records to enhance cancer surveillance. In 1996, Huff and co-authors estimated disease occurrence rates from hospital discharge data for breast, cervical and lung cancer at a state- and county level for the state of Maine, US. Consistently with our results, rates from hospital discharge data were higher than rates from cancer registry data. It is noteworthy that the breast cancer rates from NHDRs and Cancer Registry data were the ones with the higher correlation among those considered (correlation coefficients were $0.87,0.79$ and 0.55 for breast, lung and cervical cancer, respectively) [26]. We have previously proposed the use of the NHDRs to evaluate the breast cancer burden in Italy [11]. Results across our two studies are fairly consistent. However, results from our previous study were limited by the inclusion of repeat hospital admissions. Moreover, a different and more restricted time window was considered (i.e., 2000-2005). Ferretti et al. used an algorithm based on Regional hospital discharge records to estimate breast cancer incidence in three Italian regions covered by the Italian net of CRs (e.g., Emilia Romagna, Toscana and Veneto). Incidence rates of the two methods showed no statistical differences. However, the authors ascribed the agreement between hospital discharge records and CRs incidence rates to a cross effect of both sensitivity and specificity limitations of the discharge records algorithm [27].

\section{Conclusions}

A National system of population-based CRs is essential to monitor cancer patterns and trends at a National and local level and to orient health monitoring and resource allocation decisions [28]. However, the exclusive use of CRs may pose limits to the estimate of cancer burden, mainly due to incomplete and heterogeneous coverage. We suggest the use of the NHDRs to supplement the net of CRs. The latter source (NHDRs) may be a valuable and relatively efficient tool for enhancing cancer surveillance.

\section{Abbreviations}

et al: And co-authors; AAPC: Average annual percentage change; CR: Cancer registry; CRs: Cancer registries; $\mathrm{Cl}$ : Confidence interval; e.g: Exampli gratia; HDF: Hospital discharge file; i.e: Id est; ICD-9-CM: International classification of diseases, ninth revision, clinical modification; AIRTUM: Italian association of cancer registries; Ms: Mastectomies; MIAMOD: Mortality and incidence analysis model; NHDRs: National hospital discharge Records; ISTAT: National Institute for Statistics; Qs: Quadrantectomies; SEER: Surveillance, epidemiology and end results program; US: United states.

\section{Competing interests}

The authors declare that they have no competing interests.

\section{Authors' contributions}

$\mathrm{PP}$, study conception and design, data acquisition, manuscript drafting. MB, manuscript drafting, methodological advise. MC, critical revision for important intellectual contents. MDM, critical revisions for important intellectual contents. AS, critical revisions for important intellectual contents $M D$, critical revisions for important intellectual contents. AF, critical revisions for important intellectual contents. AL, critical revisions for important intellectual contents. FP, critical revisions for important intellectual contents. PM, critical revisions for important intellectual contents. GC, critical revisions for important intellectual contents; AA, critical revisions for important intellectual contents. $\mathrm{CN}$, critical revisions for important intellectual contents. $A D$, critical revisions for important intellectual contents. GDT, data analysis, results' interpretation. MLB, critical revisions for important intellectual contents. AM, critical revisions for important intellectual contents. IRM, data acquisition, methodological advise. AG, study conception and design, methodological advice. All authors read and approved the final manuscript.

\section{Acknowledgements}

This work was supported by the Human Health Foundation, Spoleto (PG), Italy (http://www.hhfonlus.org), the Sbarro Health Research Organization, Philadelphia, PA (http://www.shro.org), the DoD, Army Research and Development, and the DoH Commonwealth of Pennsylvania. Authors are also grateful to the Euro Mediterranean Scientific Institute (ISBEM, Brindisi), for data management and analysis.

\section{Author details}

'Department of Internal Medicine, University of Florence, Viale Pieraccini 18 Florence 50139, Italy. ${ }^{2}$ Scientific Direction, Regina Elena National Cancer Institute, Via Elio Chianesi 53, 00144 Rome, Italy. ${ }^{3}$ Epidemiology, Regina Elena National Cancer Institute, Via Elio Chianesi 53, 00144 Rome, Italy. ${ }^{4}$ Local Health Authority of Naples (ASL NA1), Centro piazza Nazionale 95, Naples 80143, Italy. ${ }^{5}$ Department of General Surgery, Second University of Naples, Via Sergio Pansini, Naples 80131, Italy. ${ }^{6}$ Department of Senology, National Cancer Institute G. Pascale Foundation, Via Mariano Semmola, Naples 80131, Italy. ${ }^{7}$ Sbarro Institute for Cancer Research and Molecular Medicine and Center of Biotechnology, College of Science and Technology Temple University, BioLife Science, Bldg. Suite 4311900 N 12th Street, Philadelphia, PA 19122, USA. ${ }^{8}$ Radiotherapy, Local Health Authority Salerno, via Nizza 146, Salerno 84124, Italy. ${ }^{9}$ INT-CROM, National Cancer Institute G. Pascale Foundation - Cancer Research Center, Via Ammiraglio Bianco, Mercogliano, Avellino 83013, Italy. ${ }^{10}$ Human Health Foundation, Piazza Pianciani 5, Spoleto-Perugia 06049, Italy. ${ }^{11}$ Euro Mediterranean Biomedical Institute (ISBEM) Research Centre, Via Reali di Bulgaria, Brindisi, Mesagne 72023, Italy. 
${ }^{12}$ Department of Public Health and Infectious Diseases, La Sapienza University of Rome, Piazzale Aldo Moro 5, Rome 00185, Italy. ${ }^{13}$ Department of Surgery, Jefferson Medical College, Thomas Jefferson University, Philadelphia PA 19107, USA. ${ }^{14}$ Department of Human Pathology \& Oncology, University of Siena, Strada delle Scotte, Siena 53100, Italy.

Received: 18 May 2012 Accepted: 18 June 2012

Published: 20 November 2012

\section{References}

1. Jensen OM, Whelan S: Planning a cancer registry. Danish CancerRegistry. IARC Sci Publ 1991, 95:22-28.

2. Miller M, Swan J: SEER doubles coverage by adding registries for four states. J Natl Cancer Inst 2001, 93(7):500.

3. Ellekjaer $\mathrm{H}$, Holmen J, Krüger $\mathrm{O}$, Terent A: Identification of incident stroke in Norway: hospital discharge data compared with a population-based stroke register. Stroke 1999, 30(1):56-60

4. Mähönen M, Salomaa V, Brommels M, Molarius A, Miettinen H, Pyörälä K, Tuomilehto J, Arstila M, Kaarsalo E, Ketonen M, Kuulasmaa K, Lehto S, Mustaniemi H, Niemelä M, Palomäki P, Torppa J, Vuorenmaa T: The validity of hospital discharge register data on coronary heart disease in Finland. Eur J Epidemiol 1997, 13(4):403-415.

5. Brooks JM, Chrischilles E, Scott S, Ritho J, Chen-Hardee S: Information gained from linking SEER Cancer Registry Data to state-level hospital discharge abstracts. Surveillance, Epidemiology, and End Results. Med Care 2000, 38(11):1131-1140.

6. Du X, Freeman JL, Warren JL, Nattinger AB, Zhang D, Goodwin JS: Accuracy and completeness of Medicare claims data for surgical treatment of breast cancer. Med Care 2000, 38(7):719-727.

7. Cooper GS, Yuan Z, Stange KC, Dennis LK, Amini SB, Rimm AA: Agreement of Medicare claims and tumor registry data for assessment of cancerrelated treatment. Med Care 2000, 38(4):411-421.

8. Freeman JL, Zhang D, Freeman DH, Goodwin JS: An approach to identifying incident breast cancer cases using Medicare claims data. J Clin Epidemiol 2000, 53(6):605-614.

9. Penberthy L, McClish D, Pugh A, Smith W, Manning C, Retchin S: Using hospital discharge files to enhance cancer surveillance. Am J Epidemiol 2003, 158(1):27-34

10. Map of the Italian Cancer Registries. http://www.registri-tumori.it/cms/ copertura\#.

11. Piscitelli P, Santoriello A, Buonaguro FM, Di Maio M, Iolascon G, Gimigliano F, Marinelli A, Distante A, Serravezza G, Sordi E, Cagossi K, Artioli F, Santangelo M, Fucito A, Gimigliano R, Brandi ML, Crespi M, Giordano A: Incidence of breast cancer in Italy: mastectomies and quadrantectomies performed between 2000 and 2005. J Exp Clin Cancer Res 2009, 28:86.

12. Health Italian Minister Hospital Discharge Form. http://www.salute.gov.it/ ricoveriOspedalieri/paginalnternaRicoveriOspedalieri.jsp? menu=rilevazione\&id=1232\&lingua=italiano.

13. Health Mo. Department of Quality Assessment, Management of Medical Care and Ethics. http://www.salute.gov.t//ministero/sezMinistero.jsp? label=ded\&id=307.

14. Center for Diseases Control and Prevention. http://www.cdc.gov/nchs/icd/ icd $9 \mathrm{~cm} . \mathrm{htm}$.

15. Health IMo. ICD9CM codes. http://www.salute.gov.it/ricoveriOspedalieri/ paginalnternaMenuRicoveriOspedalieri.jsp? menu=classificazione\&id=1278\&lingua=italiano

16. Giorgi D, Giordano L, Ventura L, Frigerio A, Paci E, Zappa M: Mammography screening in Italy: 2008 survey. Epidemiol Prev 2010, 34(5-6 Suppl 4):9-25.

17. Millikan R, Dressler L, Geradts J, Graham M: The need for epidemiologic studies of in-situ carcinoma of the breast. Breast Cancer Res Treat 1995, 35(1):65-77

18. Izquierdo JN, Schoenbach VJ: The potential and limitations of data from population-based state cancer registries. Am J Public Health 2000, 90(5):695-698

19. Cardoso F, Senkus-Konefka E, Fallowfield L, Costa A, Castiglione M, ESMO Guidelines Working Group: Locally recurrent or metastatic breast cancer: ESMO Clinical Practice Guidelines for diagnosis, treatment and follow-up. Ann Oncol 2010, 21(Suppl 5):v15-v19.

20. Mendlein JM, Franks AL: Hospital discharge data. Using chronic disease data: a handbook for public health practitioners. Atlanta: Centers for Disease Control and Prevention; 1992
21. Keller RB, Soule DN, Wennberg JE, Hanley DF: Dealing with geographic variations in the use of hospitals. The experience of the maine medical assessment foundation orthopaedic study group. J Bone Joint Surg Am 1990, 72(9):1286-1293.

22. AIRTUM Working Group: Cancer incidence in Italy: 2006 estimates. Epidemiol Prev 2006, 2:105-106.

23. Fisher B, Anderson S, Redmond CK, Wolmark N, Wickerham DL, Cronin WM: Reanalysis and results after 12 years of follow-up in a randomized clinical trial comparing total mastectomy with lumpectomy with or without irradiation in the treatment of breast cancer. N Engl J Med 1995, 333(22):1456-1461

24. Wapnir IL, Anderson SJ, Mamounas EP, Geyer CE Jr, Jeong JH, Tan-Chiu E, Fisher B, Wolmark N: Prognosis after ipsilateral breast tumor recurrence and locoregional recurrences in five National Surgical Adjuvant Breast and Bowel Project node-positive adjuvant breast cancer trials. J Clin Oncol 2006, 24(13):2028-2037.

25. Pálka I, Kelemen G, Ormándi K, Lázár G, Nyári T, Thurzó L, Kahán Z: Tumor characteristics in screen-detected and symptomatic breast cancers. Pathol Oncol Res 2008, 14(2):161-167.

26. Huff L, Bogdan G, Burke K, Hayes E, Perry W, Graham L, Lentzner H: Using hospital discharge data for disease surveillance. Public Health Rep 1996, 111(1):78-81.

27. Ferretti S, Guzzinati S, Zambon P, Manneschi G, Crocetti E, Falcini F, Giorgetti S, Cirilli C, Pirani M, Mangone L, Di Felice E, Del Lisi V, Sgargi P, Buzzoni C, Russo A, Paci E: Cancer incidence estimation by hospital discharge flow as compared with cancer registries data. Epidemiol Prev 2009, 4-5:14-53.

28. Parkin DM, Wagner G, Muir CS: The Role of the Registry in Cancer Control Lyon, International Agency for Research on Cancer; 1985.

\section{doi:10.1186/1756-9966-31-96}

Cite this article as: Piscitelli et al:: The burden of breast cancer in Italy: mastectomies and quadrantectomies performed between 2001 and 2008 based on nationwide hospital discharge records. Journal of Experimental \& Clinical Cancer Research 2012 31:96.

\section{Submit your next manuscript to BioMed Central and take full advantage of:}

- Convenient online submission

- Thorough peer review

- No space constraints or color figure charges

- Immediate publication on acceptance

- Inclusion in PubMed, CAS, Scopus and Google Scholar

- Research which is freely available for redistribution 